\title{
DOR E PARESTESIAS NOS MEMBROS SUPERIORES E DIAGNÓSTICO DA SÍNDROME DO TÚNEL DO CARPO
}

\author{
Valéria Ribeiro Nogueira Barbosa1 , Fábio Galvão Dantas², \\ Maria Aparecida Alves Cardoso33, Jovany Luis Alves de Medeiros ${ }^{4}$
}

\begin{abstract}
RESUMO - Nós avaliamos a freqüência e localização de dor e parestesias em pacientes com síndrome do túnel do carpo (STC) e em indivíduos da população geral, pareados por gênero e idade. Determinamos a sensibilidade e a especificidade desses sintomas para o diagnóstico de STC. Dor foi um sintoma comum nos dois grupos de pacientes. Parestesia ocorreu com mais freqüência em pacientes com STC $(p<0,05)$. No grupo com STC, a dor era localizada no pescoço $(42,8 \%)$, membros superiores $(36,8 \%)$ e mãos $(82,8 \%)$. Nos pacientes sem STC, a dor se localizava na cabeça $(11,4 \%)$, região axial $(37,1 \%)$ e membros inferiores $(22,8 \%)$. Nós concluímos que, em relação ao diagnóstico de STC, dor e parestesias apresentam sensibilidade baixa e especificidade alta quando as queixas estão presentes nos membros superiores; e sensibilidade e especificidade altas (>75\%) quando são localizadas nas mãos.
\end{abstract}

PALAVRAS-CHAVE: síndrome do túnel do carpo, nervo mediano, diagnóstico, eletroneurografia, dor, parestesias.

\begin{abstract}
Pain and numbness in the arms and hands and carpal tunnel syndrome diagnosis
ABSTRACT - We studied the frequency and localization of pain and numbness in patients with carpal tunnel syndrome (CTS), in comparison with individuals of the general population, matched for sex and age, and we determined the sensitivity and the specificity of these symptoms for the CTS diagnosis. Pain was a common symptom in the two groups of patients. Numbness occurred more frequently in CTS group $(p<0.05)$. In CTS patients, pain complaints were present in neck $(42.8 \%)$, arms $(36.8 \%)$ and hands $(82.8 \%)$. Among controls, pain was more common in head $(11.4 \%)$, trunk $(37.1 \%)$, legs $(22.8 \%)$. In our casuistics, in relation to the CTS diagnosis, the presence of pain and numbness have low sensitivity and high specificity when they occur in the arms, and high sensitivity and specificity when they occur in the hands.
\end{abstract}

KEY WORDS: carpal tunnel syndrome, median nerve, conduction nerve, pain and numbness, diagnosis.

A síndrome do túnel do carpo (STC) é patologia freqüente ${ }^{1,2}$, que se expressa por dor e parestesias nas mãos, comumente em pacientes com idades entre 30 e 50 anos $^{3-5}$. Quase sempre, não há dificuldade diagnóstica nos casos típicos.

No entanto, há incerteza em muitos pacientes, com implicações clínicas, jurídicas e sociais, possivelmente porque inexiste um critério clínico ideal para o diagnóstico ${ }^{3}$, pois os dados da maioria dos estudos são derivados de pacientes atendidos em serviços especializados $^{6-9}$, o que significa que os sintomas descritos são comumente de pacientes com sintomatologia mais grave, que já procuraram assistência médica.

Neste estudo, nós objetivamos determinar a freqüência e a localização de dor e parestesias em pa- cientes com STC, e em pessoas da população geral, pa reados por gênero e idade, assim como calculamos a sensibilidade e a especificidade desses sintomas em relação ao diagnóstico de STC.

\section{MÉTODO}

Realizamos estudo comparativo, com dois grupos. O g rupo experimental foi constituído por pacientes que apresentavam características clínicas de STC, sobretudo a presença de parestesia noturna que melhorava com movimento vigo roso da mão como quem agita um term ômetro, sinal da sacudida (flick sign ${ }^{3}$ ) e alterações nos estudos de condução nervosa típicas para esse diagnóstico. $\mathrm{O}$ grupo controle foi composto por pessoas abordadas ao acaso, em lugares públicos da cidade de Campina Grande - PB. Quando elas pe rmitiam a sua participação, tinham a mesma idade e eram

Departamento de Fisioterapia da Universidade Estadual da Paraíba - Campus I - Campina Grande PB, Brasil (UEPB): ${ }^{1}$ Professora de Fisioterapia, Mestre em Saúde Coletiva, Departamento de Fisioterapia UEPB; ${ }^{2}$ Professor de Neurologia, Mestre em Neurologia, Departamento de Fisioterapia da UEPB; ${ }^{3}$ PhD, Professor de Epidemiologia Doutor, Departamento de Farmácia e Bioquímica, UEPB; ${ }^{4}$ Professor de Neurologia Doutor, Departamento de Fisioterapia, UEPB.

Recebido 15 Dezembro 2005, recebido na forma final 25 Julho 2006. Aceito 13 Setembro 2006.

Dr. Jovany Luis Alves de Medeiros - Rua João Alves de Lira 1136 / 1002 - 58101-281 Campina Grande PB - Brasil.

E-mail: jovany_medeiros@hotmail.com 
do mesmo gênero de um dos pacientes do grupo experimental e foram incluídos no protocolo. Todos os pacientes responderam a interrogatório sintomatológico sobre os diversos sistemas, não se encontrando nada relevante, e os pacientes com STC se submeteram a exame neurológico, realizado por dois dos autores (VRNB e JLAM).

O estudo da velocidade de condução nervosa foi realiza do com o paciente deitado. As amplitudes dos potenciais registrados foram medidas pico a pico e as latências medidas no início da fase negativa do potencial de ação. A estimulação elétrica, realizada com eletrodo bipolar, com o catodo sobrea prega proximal do punho, teve a mesma localização tanto para o estudo da condução nervosa motora quanto sensitiva. Aumentou-se prog ressivamente a intensidade do estímulo elétrico até uma resposta máxima ser obtida, então se estimulou o nervo com uma intensidade de $20 \%$ acima do estímulo máximo. $O$ eletrodo terra foi fixado na região dorsal do punho.

Os potenciais de ação de nervos sensitivos (PANS) foram registrados por método antidrômico com eletrodo de anel, G1 na articulação metacarpiana e G2 na articulação interfalangeana proximal no segundo, terce i ro e quarto dedos para o nervo mediano e quarto dedos para o nervo ulnar. A distância entre o eletrodo de estímulo e registro foi, em média, de $12 \mathrm{~cm}$.

Os potenciais de ação muscular compostos (PAMC) foram registrados com eletrodo de superfície, fixados sobre os músculos da região tenar (G1) e polegar (G2). Estimulouse eletricamente o nervo mediano no punho e cotovelo. A distância entre os estímulos distal e proximal foi, em média, $24 \mathrm{~cm}$. O diagnóstico neurofisiológico de STC foi estabelecido quando a latência do PANS, ou do PAMC, obtido após estimulação do nervo mediano no punho, era maior do que 2,6 ms e 4,2 ms, respectivamente; ou quando a latência do PANS registrado no nervo mediano, no segmento punhoIV dedo, era igual ou superior a 0,4 ms em relação ao PANS obtido no nervo ulnar no mesmo segmento. A temperatura da mão foi medida antes e após o teste com termômetro digital, devendo-se manter acima de $30^{\circ} \mathrm{C}$ nas duas medições para validação dos resultados. Os testes neurofisiológicos foram realizados por um dos autores (JLAM).

Todos os pacientes responderam a quatro perguntas: se sentiam alguma dor? Em caso afirmativo, em que parte do corpo? Se sentiam alguma dormência? Em caso afirm ativo, em que parte do corpo? As respostas foram analisadas e a presença de dor e parestesias nos dois grupos de pacientes foram comparadas com o teste do qui-quadrado. Utiliza- mos o teste $t$ de Student para comparar as médias de idade entre os dois grupos de pacientes.

A pesquisa foi aprovada pelo Comitê de Ética da Universidade Estadual da Paraíba, protocolo n 013/02.

\section{RESULTADOS}

Casuística - Entre agosto e dezembro de 2002, 35 pacientes (34 mulheres e um homem) idades entre 34 e 72 anos, média 51 \$9,8 anos, foram encaminhados para o laboratório de Neurofisiologia da Clínica Santa Maria, em Campina Grande - PB, para re alização de exame eletroneuromiográfico, com suspeita de STC. Eles tiveram o diagnóstico confirmado pela eletroneurografia. Outros 35 pacientes, 34 mulheres e um homem, idades entre 33 e 72 anos, média $51 \pm$ 9,7 anos, foram abordados ao acaso, na mesma cidade, e constituíram o grupo controle. As características dos pacientes dos dois grupos foram similares no que diz respeito a gênero e idade.

Todos os pacientes com STC apresentavam dor ou p a restesia, ou os dois sintomas conjuntamente, nos membros superiores (MMSS) ou nas mãos (Tabela 1). A localização da dor e da parestesia foi diferente entre os pacientes com e sem STC.

Dor - Não houve diferença significativa em relação à freqüência da dor, nos dois grupos de pacientes. Houve maior variabilidade na localização da dor entre os pacientes do grupo controle, onde predominou dor de cabeça, dor na região axial e nos membros inferiores (CMMII) (Tabela 2). Entre os pacientes com STC, a localização da dor foi mais freqüente no pescoço e nos MMSS. Apenas seis (17\%) pacientes com STC não se queixavam de dor. A sensibilidade para dor nos MMSS, em relação ao diagnóstico de STC, foi $40 \%$, com especificidade de $94,3 \%$. Para dor nas mãos, a sensibilidade e especificidade foram $82,8 \%$ e $91,4 \%$, respectivamente.

Pa restesia - Como esperado, houve maior prevalência de parestesia entre os pacientes com STC $(p<$ $0,05)$. No grupo controle, seis pacientes $(17 \%)$ relata-

Tabela 1. Respostas à pergunta: você apresenta alguma dor ou dormência em alguma parte do corpo? Em pacientes com e sem STC.

\begin{tabular}{lccccc}
\hline Pacientes & \multicolumn{2}{c}{ Dor } & & \multicolumn{2}{c}{ Parestesia } \\
\cline { 2 - 3 } \cline { 6 - 6 } & Sim & Não & & Sim & Não \\
\hline Com STC n (\%) & $29(83 \%)$ & $6(17 \%)$ & & $30(86 \%)$ & $5(14 \%)$ \\
Sem STC n (\%) & $24(69 \%)$ & $11(31 \%)$ & & $6(17 \%)$ & $29(83 \%)$ \\
\hline
\end{tabular}

Teste do qui quadrado $(p<0,05)$. 
Tabela 2. Lcalização dos sintomas dor e parestesias entre 35 pacientes com STC e 35 pacientes sem STC.

\begin{tabular}{|c|c|c|c|c|c|}
\hline \multicolumn{2}{|c|}{ Pacientes } & \multicolumn{2}{|c|}{ Com STC } & \multicolumn{2}{|c|}{ Sem STC } \\
\hline Sintomas & Localização* & $\mathrm{N}$ & $\%$ & $\mathrm{~N}$ & $\%$ \\
\hline \multirow[t]{7}{*}{ Dor } & Cabeça & 0 & 0 & 4 & 11,4 \\
\hline & Pescoço & 15 & 42,8 & 2 & 5,7 \\
\hline & Região axial & 6 & 17,1 & 13 & 37,1 \\
\hline & MMSS & 14 & 36,8 & 2 & 5,7 \\
\hline & Mãos & 29 & 82,8 & 3 & 8,5 \\
\hline & MMII & 3 & 8,6 & 8 & 22,8 \\
\hline & dor radicular & 6 & 17,1 & 0 & 0 \\
\hline \multirow[t]{2}{*}{ Parestesia } & MMSS & 8 & 14 & 0 & 0 \\
\hline & Mãos & 27 & 77 & 6 & 17,1 \\
\hline
\end{tabular}

Teste o qui quadrado $(\mathrm{p}<0,05) ; \mathrm{STC}$, síndrome do túnel do carpo; MMII, membros inferiore; MMSS, membros superiores. *A localização dos sintomas tem valor maior do que o número de pacientes porque um mesmo paciente poderia referir mais de uma segmento afetado.

ram parestesia, que era localizada nos MMSS (17\%). Em relação ao diagnóstico de STC parestesia nas mãos teve sensibilidade de $77,1 \%$ e especificidade de $82,8 \%$.

\section{DISCUSSÃO}

O nosso estudo trata do diagnóstico de STC, um tema sempre relevante dado às incertezas e a presença de quantidade não desprezível de diagnósticos falsos positivos e falsos negativos na prática diária. A nosso ver, o critério diagnóstico para STC é um problema ainda a ser definido. O risco para o especialista é que, apoiado em informações quase sempre surgidas em análises de casos provenientes de serviços especializados ${ }^{6-9}$, sinta a confortável sensação de que já não existam perguntas a serem respondidas sobre o tema.

Sintomas compatíveis com STC ocorrem na população geral ${ }^{2,10}$; no nosso estudo, seis (17\%) dos nossos pacientes do grupo controle relataram parestesias nos MMSS, esse fato é previsto pelo teorema das probabilidades condicionadas de Bayes, segundo o qual a freqüência de sintomas falsos positivos em uma população depende não apenas do valor do teste diagnóstico aplicado, mas da prevalência da doença nessa população ${ }^{11}$.

Dor tem alta freqüência na população geral ${ }^{12-15}$, sendo considerado o mais comum sintoma presente na prática clínica ${ }^{16}$, fato esse corroborado no nosso g rupo controle, visto que $69 \%$ dos pacientes afirm a- ram ser portadores de algum tipo de dor. É possível que esse número reflita a abordagem que adotamos: uma pergunta direta que favorece uma resposta afirmativa. Em relação à dor, o fator diferencial entre os pacientes com STC e sem STC foi a sua localização: maior freqüência dor nas mãos nos pacientes com STC e dor de cabeça para os pacientes do grupo controle.

Vários autores determinaram a sensibilidade e especificidade dos testes provocativos no diagnóstico de STC ${ }^{9,17-19 ;}$ no entanto, dor e parestesia nas mãos são descritos como sintomas importantes para o diagnóstico sem que os valores desses índices tenham sido ainda calculados. Na nossa amostra, em relação ao diagnóstico de STC, dor e parestesias nas mãos têm sensibilidade e especificidade alta $(>75 \%)$ e dor nos MMSS tem sensibilidade baixa $(<40 \%)$ e especificidade alta.

\section{REFERÊNCIAS}

1. A t roshi I, Gummesson C, Johnsson R, Ornstein E, Ranstan J, Rosén I. Prevalence of carpal tunnel syndrome in a general population. JAMA 1999;282:153-158.

2. Mondelli M, Giannini F, Giacchi M. Carpal tunnel syndrome incidence in a general population. Neurology 2001;58:289-294.

3. D'Arcy CA, McGee S.The rational clinical examination: does this patient have carpal tunnel syndrome? JAMA 2000;21,283:3110-3117.

4. Gupta SK, Benstead TJ. Symptoms experienced by patients with carpal tunnel syndrome. Can J Neurol Sci 1997;24:338-342.

5. Dawson DM, Hallet M, Wilbourn AJ. Carpal tunnel syndrome. In Entrapment neuropathies. $3^{\text {rd }}$ ed. Philadelphia: Lippincott-Raven, 1999:20-94.

6. Kouyoumdjian JA. Síndrome do túnel do carpo: aspectos clínico-epidemiológicos em 668 casos. Arq Neuropsiquiatr 1999;57:202-207. 
7. Campos CC, Manzano GM, Castelo Filho A, Nóbrega JAM. Parestesia e/ou dor nas mãos e/ou punhos como motivo de encaminhamento para estudo eletron eu romiográfico. A rq Neuropsiquiatr 2003;61:56-60.

8. Stevens JC, Smith BE, Weaver AL, Bosch EP, Deen HG, Wilkens JA. Symptoms of 100 patients with electromyographically verified carpal tunnel syndrome. Muscle Nerve 1999;22:1448-1456.

9. Pryse-Phillips WE. Validation of a diagnostic sign in carpal tunnel syndrome. J Neurol Neurosurg Psychiatry 1984;47:870-872.

10. Tanaka S, Wild DK, Seligman PJ, Behrens V, Cameron L, Putz-Anderson, V. The US prevalence of self-reported carpal tunnel syndrome: 1988 National Health Interview Survey Data. Am J Pub Health 1994;84:18461848.

11. Yudkowsky E. An intuitive explanation of bayesian reasoning. http:/ / yudkowsky.net/ bayes/bayes.html. Acesso em 06/junho/2006.

12. Hessel A, Geyer M, Gunzelmann T, Schumacher J, Brahler EZ. Somatoform complaints in elderly of Germany. Gerontol Geriatr 2003;36: 287-296.
13. Al-Arfaj AS, Alballa SR, Al-Dalaan AN, et al. Musculoskeletal pain in the community. Saudi Med J 2003;24:863-867.

14. Peltonen M, Lindroos AK, Torgerson JS. Musculoskeletal pain in the obese: a comparison with a general population and long-term changes after conventional and surgical obesity treatment. Pain 2003;104:549-555.

15. Abusdal UG, Hagen KB, Bjorndal A. Self-reported chronic muscle pain among women in Oslo. Tidsskr Nor Laegeforen 1997;117:1606-1610.

16. No ren J, Frazier T, Altman I, DeLozier J. Ambulatory medical care: a comparasion in internists, and family-general practitioners. N Eng J Med 1980;302:11-16.

17. Golding DN, Rose DM, Selvarajah K. Clinical tests for carpal tunnel syndrome: an evaluation. Br J Rheumatol 1986;25:388-390.

18. Katz JN, Larson MG, Sabra A, et al. The carpal tunnel syndrome: diagnostic utility of the history and physical examination findings. Ann Intern Med 1990;112:321-327.

19. Stewart JD, Eisen A. Tinel's sign and the carpal tunnel syndrome. Br Med J 1978;2:1125-1126. 